\title{
Monotone positive solution of nonlinear third-order two-point boundary value problem
}

Yongping Sun, Min Zhao, and Shuihong Li 


\title{
MONOTONE POSITIVE SOLUTION OF NONLINEAR THIRD-ORDER TWO-POINT BOUNDARY VALUE PROBLEM
}

\author{
YONGPING SUN, MIN ZHAO, AND SHUHONG LI
}

Received 18 September, 2012

\begin{abstract}
In this paper, we are concerned with the existence of monotone positive solution to the nonlinear third-order two-point boundary value problem

$$
\left\{\begin{array}{l}
x^{\prime \prime \prime}(t)+f(x(t))=0, \quad t \in(0,1), \\
x(0)=x^{\prime \prime}(0)=x^{\prime}(1)=0 .
\end{array}\right.
$$

Under suitable assumptions on $f$ and by using a fixed point theorem of cone expansion and compression of functional type due to Avery, Anderson and Krueger, we establish the existence of at least one positive solution of the boundary value problem.
\end{abstract}

2010 Mathematics Subject Classification: 34B14; 34B15; 34B18

Keywords: monotone positive solution, nonlinear boundary value problem, fixed point theorem, existence

\section{INTRODUCTION}

In this paper, we are concerned with the existence of single monotone positive solution to nonlinear third-order two-point boundary value problem (BVP):

$$
\left\{\begin{array}{l}
x^{\prime \prime \prime}(t)+f(x(t))=0, \quad t \in(0,1), \\
x(0)=x^{\prime \prime}(0)=x^{\prime}(1)=0,
\end{array}\right.
$$

where $f:[0, \infty) \rightarrow[0, \infty)$ is continuous. Here, by a positive solution $u^{*}$ of BVP (1.1), we mean a function $u^{*}$ satisfies (1.1) and $u^{*}(t)>0$, for $0<t<1$.

Third-order equations arise in a variety of different areas of applied mathematics and physics, such as the deflection of a curved beam having a constant or varying cross section, three layer beam, electromagnetic waves or gravity-driven flows and so on [17]. The existence of positive solutions of third-order equations with two-point or three-point boundary conditions have been studied by several authors using the fixed point index theorem, Leray-Schauder continuation theorem, nonlinear alternative of Leray, upper solution and lower solutions method, and comparison principles and so

Project was supported by Natural Science Foundation of Zhejiang Province of China (LY12A01012).

(c) 2014 Miskolc University Press 
on. One may see $[3,4,7-11,13-16,18,20-24,26]$ and the references therein for some related results. In a recent paper [25], by applying a monotone iterative method, Sun studied the boundary value problem

$$
\left\{\begin{array}{l}
x^{\prime \prime \prime}(t)+f\left(t, x(t), x^{\prime}(t)\right)=0, \quad t \in(0,1), \\
x(0)=x^{\prime \prime}(0)=x^{\prime}(1)=0,
\end{array}\right.
$$

where $f:[0,1] \times[0, \infty) \times[0, \infty) \rightarrow[0, \infty)$ is continuous, and $q:(0,1) \rightarrow[0, \infty)$ is a continuous function such that $0<\int_{0}^{1}(1-s) q(s) d s<\infty$. The iterative schemes for approximating the solutions are obtained.

One of the most frequently used tools for proving the existence of positive solutions to the boundary value problems is Krasnosel'skii's theorem on cone expansion and compression and its norm-type version due to Guo [19]. Many authors have used this approach or a variation to obtain positive solution of boundary value problem; see [1,2]. Recently, Anderson and Avery in [5], Avery, Anderson and Krueger in [6] generalized the fixed point theorem of cone expansion and compression of norm type by replacing the norms with two functionals satisfying certain conditions to produce a fixed point theorem of cone expansion and compression of functional type, and they applied the fixed point to verify the existence of one positive solution to a discrete second-order conjugate boundary value problem and the right focal second order boundary value problem. Ehrke [12] applied this fixed point to verify the existence of one positive solution to the left focal second order boundary value problem. According to [5], the flexibility of using functionals instead of norms allows the theorem to be used in a wider variety of situations especially in applications to boundary value problems it allows for improved sufficiency conditions for the existence of a positive solution.

Motivated greatly by the above-mentioned works, in this paper we will try using this fixed point theorem to consider the existence of monotone positive solution to BVP (1.1). This paper is organized as follows. In Section 2, we present some definitions and background results on cones and completely continuous operators. We also state the fixed point theorem of cone expansion and compression of functional type. Some lemmas and the main result will be given in Section 3. In Section 4, an explicit example is given to illustrate our main result.

\section{PRELIMinaries}

In this section, we present some definitions and background results on cones and completely continuous operators. We also state the fixed point theorem of cone expansion and compression of functional type. 
Definition 1. Let $E$ be a real Banach space. A nonempty closed convex set $P \subset E$ is called a cone of $E$ if it satisfies the following two conditions:

(1) $x \in P, \lambda>0$ implies $\lambda x \in P$;

(2) $x \in P,-x \in P$ implies $x=0$.

Every cone $P \subset E$ induces an ordering in $E$ given by $x \leq y$ if and only if $y-x \in$ $P$.

Definition 2. A cone $P$ of a real Banach space $E$ is said to be normal if there exists $\delta>0$ such that $\|x+y\| \geq \delta$ for all $x, y \in P$ with $\|x\|=\|y\|=1$.

It is well known that for a cone $P$ in a real Banach space $E, P$ is normal if and only if the norm of the Banach space $E$ is semimonotone. That is, there exists a constant $N>0$ such that $0 \leq x \leq y$ implies that $\|x\| \leq N\|y\|$. A proof of this result can be found in [19].

Definition 3. Let $E$ be a real Banach space. An operator $T: E \rightarrow E$ is said to be completely continuous if it is continuous and maps bounded sets into precompact sets. Additionally, the operator $T$ is called increasing on a domain $D$, provided that $T x_{1} \leq T x_{2}$ for all $x_{1}, x_{2} \in D$ with $x_{1} \leq x_{2}$.

Let $\alpha$ and $\gamma$ are non-negative continuous functionals on $P$; then, for positive real numbers $r$ and $R$, we define the following sets:

$$
\begin{gathered}
P(\alpha, r)=\{x \in P: \alpha(x)<r\}, \\
P(\gamma, R)=\{x \in P: \gamma(x)<R\}, \\
P(\gamma, \alpha, r, R)=\{x \in P: r<\alpha(x) \text { and } \gamma(x)<R\} .
\end{gathered}
$$

Now we state the fixed point theorem of cone expansion and compression of functional type due to Anderson, Avery, and Krueger in [6].

Theorem 1. Let $P$ be a normal cone in a real Banach space $E$, and let $\alpha$ and $\gamma$ are nonnegative continuous functionals on $P$. Assume $P(\gamma, \alpha, r, R)$ as above is a nonempty bounded subset of $P$,

$$
T: \overline{P(\gamma, \alpha, r, R)} \rightarrow P
$$

is a completely continuous operator and

$$
\overline{P(\alpha, r)} \subseteq P(\gamma, R) .
$$

If one of the two conditions,

(H1) $\alpha(T x) \leq r$ for all $x \in \partial P(\alpha, r), \gamma(T x) \geq R$ for all $x \in \partial P(\gamma, R)$,

$$
\inf _{x \in \partial P(\gamma, R)}\|T x\|>0,
$$

and for all $y \in \partial P(\alpha, r), z \in \partial P(\gamma, R), \lambda \geq 1$, and $\mu \in(0,1]$, the functionals satisfy the properties

$$
\alpha(\lambda y) \geq \lambda \alpha(y), \gamma(\mu z) \leq \mu \gamma(z), \text { and } \alpha(0)=0,
$$


(H2) $\alpha(T x) \geq r$, for all $x \in \partial P(\alpha, r), \gamma(T x) \leq R$, for all $x \in \partial P(\gamma, R)$,

$$
\inf _{x \in \partial P(\alpha, r)}\|T x\|>0
$$

and for all $y \in \partial P(\alpha, r), z \in \partial P(\gamma, R), \lambda \in(0,1]$, and $\mu \geq 1$, the functionals satisfy the properties

$$
\alpha(\lambda y) \leq \lambda \alpha(y), \gamma(\mu z) \geq \mu \gamma(z), \text { and } \gamma(0)=0,
$$

is satisfied, then $T$ has at least one fixed point $x^{*}$ such that

$$
r \leq \alpha\left(x^{*}\right) \text { and } \gamma\left(x^{*}\right) \leq R .
$$

Moreover, suppose there exist $x_{l}, x_{u} \in P$ such that $\overline{P(\gamma, \alpha, r, R)} \subseteq\left[x_{l}, x_{u}\right]$.

(E1) If there exists an increasing, completely continuous operator $U:\left[x_{l}, x_{u}\right] \rightarrow$ $P$, such that $T x \leq U x$ for all $x \in\left[x_{l}, x_{u}\right]$ and $U^{2} x_{u} \leq U x_{u}$, then

$$
x^{*} \leq x_{u}^{*} \leq U^{n} x_{u},
$$

where $n \in N$ and $x_{u}^{*}=\lim _{n \rightarrow \infty} U^{n} x_{u}$.

(E2) If there exists an increasing, completely continuous operator $L:\left[x_{l}, x_{u}\right] \rightarrow$ $P$, such that $L x \leq T x$ for all $x \in\left[x_{l}, x_{u}\right]$ and $L x_{l} \leq L^{2} x_{l}$, then

$$
L^{n} x_{l} \leq x_{l}^{*} \leq x^{*},
$$

where $n \in N$ and $x_{l}^{*}=\lim _{n \rightarrow \infty} L^{n} x_{l}$.

\section{MAIN RESULT}

In this section, we discuss the existence of positive solution of BVP (1.1). We shall consider the Banach space $E=C[0,1]$ equipped with norm $\|u\|=\max _{0 \leq t \leq 1}|u(t)|$. Denote

$$
C^{+}[0,1]=\{u \in C[0,1]: u(t) \geq 0, t \in[0,1]\} .
$$

In arriving our result, we need the following three preliminary lemmas.

Lemma 1 ([23]). Let $x(t) \in C[0,1]$, then the $B V P$ :

$$
\left\{\begin{array}{l}
u^{\prime \prime \prime}(t)+f(x(t))=0, \quad 0 \leq t \leq 1, \\
u(0)=u^{\prime \prime}(0)=u^{\prime}(1)=0,
\end{array}\right.
$$

has a unique solution

$$
u(t)=\int_{0}^{1} G(t, s) f(x(s)) d s,
$$

where

$$
G(t, s)=\left\{\begin{array}{l}
t(1-s), \quad 0 \leq t \leq s \leq 1, \\
t(1-s)-\frac{1}{2}(t-s)^{2}, \quad 0 \leq s \leq t \leq 1 .
\end{array}\right.
$$

By simple computation we can get the following results. 
Lemma 2. For any $\eta \in(0,1)$, the Green's fuction $G(t, s)$ defined by (3.2) have the following properties:

Particularly,

$$
\begin{gathered}
\int_{0}^{1} G(t, s) d s=-\frac{1}{6} t^{3}+\frac{1}{2} t, \quad t \in[0,1] ; \\
\int_{\eta}^{1} G(t, s) d s=\left\{\begin{array}{l}
\frac{1}{2}(1-\eta)^{2} t, \quad t \in[0, \eta], \\
-\frac{1}{6}(t-\eta)^{3}+\frac{1}{2}(1-\eta)^{2} t, \quad t \in[\eta, 1] .
\end{array}\right.
\end{gathered}
$$

$$
\int_{0}^{1} G(1, s) d s=\frac{1}{3}, \quad \int_{\eta}^{1} G(\eta, s) d s=\frac{1}{2} \eta(1-\eta)^{2} .
$$

Define the cone $P$ by

$$
P=\left\{x \in C^{+}[0,1]: x(t) \text { is concave and nondecreasing }\right\} .
$$

Then $P$ is a normal cone of $E$. Define the integral operator $T: P \rightarrow C^{+}[0,1]$ by

$$
(T x)(t)=\int_{0}^{1} G(t, s) f(x(s)) d s .
$$

By Lemma 1, BVP (1.1) has a positive solution $x^{*}=x^{*}(t)$ if and only if $x^{*}$ is a fixed point of $T$.

Lemma 3. For any $x \in P, \eta \in(0,1)$, the following properties hold:

(1) $x(t) \geq t\|x\|, t \in[0,1]$;

(2) $x(t) \geq \eta\|x\|, t \in[\eta, 1]$

(3) $\|x\|=x(1)$.

Lemma 4. Suppose that $f \in C([0, \infty),[0, \infty))$, then $T: P \rightarrow P$ is completely continuous.

Proof. For $x \in P$, let $w(t)=(T x)(t)$. Then $w(0)=w^{\prime \prime}(0)=w^{\prime}(1)=0$ and $w^{\prime \prime \prime}(t)=-f(x(t)) \leq 0$. Hence $w^{\prime \prime}(t)$ is decreasing and since $w^{\prime \prime}(0)=0$, we know $w^{\prime \prime}(t) \leq 0$ for all $t \in[0,1]$, thus $w(t)$ is concave and $w^{\prime}(t)$ is decreasing. By the boundary conditions $w^{\prime}(1)=0$ we have $w^{\prime}(t) \geq 0$ for all $t \in[0,1]$, thus $w(t)$ is increasing and $w(t) \geq 0$ by the boundary condition $w(0)=0$. Hence $w(t) \in P$. So $T: P \rightarrow P$. The operator $T$ is completely continuous by an application of AscoliArzela Theorem (see [27]).

Define continuous functionals $\alpha, \gamma: P \rightarrow[0, \infty)$ by

$$
\alpha(x):=\min _{t \in[\eta, 1]} x(t)=x(\eta) \text {, and } \gamma(x):=\max _{t \in[0,1]} x(t)=\|x\|=x(1) .
$$

It is clear that

$$
\alpha(\lambda y)=\lambda \alpha(y), \gamma(\mu z)=\mu \gamma(z), \text { and } \gamma(0)=0,
$$


for all $y \in \partial P(\alpha, r), z \in \partial P(\gamma, R), \lambda \in(0,1]$, and $\mu \geq 1$.

Now we discuss the existence of at least one monotone positive solution for the BVP (1.1). We obtain the following existence result.

Theorem 2. Suppose that $f \in C([0, \infty),[0, \infty)), \eta \in(0,1)$ and there exist two positive real numbers $r, R$ with $r<\eta R$ such that the following conditions are satisfied:

(A1) $f(w) \geq \frac{2 r}{\eta(1-\eta)^{2}}, \quad$ for $w \in[r, R]$,

(A2) $f(w) \leq 3 R$, for $w \in[0, R]$.

Then BVP (1.1) has at least one monotone positive solution $x^{*} \in P$ such that

$$
\frac{r t}{\eta} \leq x^{*}(t) \leq \frac{1}{2}\left(-t^{3}+3 t\right) R, \quad t \in[0, \eta],
$$

and

$$
\left(-\frac{(t-\eta)^{3}}{3 \eta(1-\eta)^{2}}+\frac{t}{\eta}\right) r \leq x^{*}(t) \leq \frac{1}{2}\left(-t^{3}+3 t\right) R, \quad t \in[\eta, 1] .
$$

Proof. From Lemma 4 we know $T: P \rightarrow P$ is completely continuous. Also, the cone $P$ is normal since the norm of the Banach space $E$ is semi-monotone. We verify a series of claims for the result.

Claim 1. $\overline{P(\alpha, r)} \subset P(\gamma, R)$.

Let $x \in \overline{P(\alpha, r)}$, from Lemma 3 we have

$$
r \geq \alpha(x)=x(\eta) \geq \eta\|x\|=\eta \gamma(x) .
$$

Thus $R>\frac{1}{\eta} r \geq \gamma(x)$, i.e. $x \in P(\gamma, R)$, Therefore, $\overline{P(\alpha, r)} \subset P(\gamma, R)$.

Claim 2. For $x \in \partial P(\alpha, r)$ (i.e. $\alpha(x)=x(\eta)=r$ ) we have $\alpha(T x) \geq r$.

Let $x \in \partial P(\alpha, r)$, then $x(s) \geq \alpha(x)=r, s \in[\eta, 1]$. Thus it follows from $(A 1)$ and Lemma 2,

$$
\begin{aligned}
\alpha(T x) & =\min _{t \in[\eta, 1]}(T x)(t)=(T x)(\eta)=\int_{0}^{1} G(\eta, s) f(x(s)) d s \geq \int_{\eta}^{1} G(\eta, s) f(x(s)) d s \\
& \geq \frac{2 r}{\eta(1-\eta)^{2}} \int_{\eta}^{1} G(\eta, s) d s=\frac{2 r}{\eta(1-\eta)^{2}} \cdot \frac{\eta(1-\eta)^{2}}{2}=r .
\end{aligned}
$$

The above arguments also yield

$$
\inf _{x \in \partial P(\alpha, r)}\|T x\| \geq r>0 .
$$


Claim 3. For $x \in \partial P(\gamma, R)$ (i.e. $\gamma(x)=x(1)=R$ ) we have $\gamma(T x) \leq R$.

Let $x \in \partial P(\gamma, R)$, then $x(s) \leq \gamma(x)=R, s \in[0,1]$. Thus from (A2) and Lemma 2 we have

$$
\begin{aligned}
\gamma(T x) & =\max _{t \in[0,1]}(T x)(t)=(T x)(1)=\int_{0}^{1} G(1, s) f(x(s)) d s \\
& \leq 3 R \int_{0}^{1} G(1, s) d s=3 R \cdot \frac{1}{3}=R .
\end{aligned}
$$

By Theorem 1 we know that $T$ has a fixed point $x^{*} \in P$ such that

$$
r \leq \alpha\left(x^{*}\right) \quad \text { and } \quad \gamma\left(x^{*}\right) \leq R
$$

Now define the increasing completely continuous operators $L: P \rightarrow P$ and $U$ : $P \rightarrow P$ by

$$
(L x)(t):=\int_{\eta}^{1} G(t, s) \frac{2 r}{\eta(1-\eta)^{2}} d s
$$

and

$$
(U x)(t):=\int_{0}^{1} G(t, s) 3 R d s
$$

respectively. Then for all $x \in P(\gamma, \alpha, r, R)$, we have

$$
L x \leq T x \leq U x
$$

Moreover, if we define $x_{l}, x_{u} \in P$ by

$$
x_{l}(t):=\left\{\begin{array}{cl}
\frac{r t}{\eta}, & t \in[0, \eta], \\
r, & t \in[\eta, 1],
\end{array} \text { and } x_{u}(t):=R, \quad t \in[0,1]\right.
$$

then as a consequence of the concavity of $P$, we have $P(\gamma, \alpha, r, R) \subset\left[x_{l}, x_{u}\right]$. Clearly, by the the definition of $U$ and $L$, for any $t \in[0,1]$, we have

$$
\left(U x_{u}\right)(t)=\int_{0}^{1} G(t, s) 3 R d s \leq 3 R \int_{0}^{1} G(1, s) d s=R=x_{u}(t)
$$


and

$$
\begin{aligned}
\left(L x_{l}\right)(t) & =\int_{\eta}^{1} G(t, s) \frac{2 r}{\eta(1-\eta)^{2}} d s=\frac{2 r}{\eta(1-\eta)^{2}} \int_{\eta}^{1} G(t, s) d s \\
& =\frac{2 r}{\eta(1-\eta)^{2}} \begin{cases}\frac{1}{2}(1-\eta)^{2} t, & t \in[0, \eta], \\
-\frac{1}{6}(t-\eta)^{3}+\frac{1}{2}(1-\eta)^{2} t, \quad t \in[\eta, 1]\end{cases} \\
& \geq \frac{2 r}{\eta(1-\eta)^{2}} \begin{cases}\frac{1}{2}(1-\eta)^{2} t, & t \in[0, \eta], \\
\frac{1}{2} \eta(1-\eta)^{2}, & t \in[\eta, 1]\end{cases} \\
& = \begin{cases}\frac{r t}{\eta}, & t \in[0, \eta], \\
r, & t \in[\eta, 1]\end{cases}
\end{aligned}
$$

This gives

$$
\left(L x_{l}\right)(t)=\frac{2 r}{\eta(1-\eta)^{2}} \int_{\eta}^{1} G(t, s) d s \leq x^{*}(t) \leq 3 R \int_{0}^{1} G(t, s) d s=\left(U x_{u}\right)(t) .
$$

It follows from Lemma 2,

$$
\frac{r t}{\eta} \leq x^{*}(t) \leq \frac{1}{2}\left(-t^{3}+3 t\right) R, \quad t \in[0, \eta],
$$

and

$$
\left(-\frac{(t-\eta)^{3}}{3 \eta(1-\eta)^{2}}+\frac{t}{\eta}\right) r \leq x^{*}(t) \leq \frac{1}{2}\left(-t^{3}+3 t\right) R, \quad t \in[\eta, 1] .
$$

This completes the proof.

\section{EXAMPLE}

In this section, in order to illustrate our result, we consider one simple example.

Example 1. Consider the BVP

$$
\left\{\begin{array}{l}
x^{\prime \prime \prime}(t)+\frac{1}{64} x^{2}(t)+\frac{1}{8} x(t)+\frac{32 \sqrt[3]{x(t)}}{1+\sqrt[3]{x(t)}}=0, \quad t \in(0,1), \\
x(0)=x^{\prime \prime}(0)=x^{\prime}(1)=0 .
\end{array}\right.
$$

Set $f(x)=\frac{1}{64} x^{2}+\frac{1}{8} x+\frac{32 \sqrt[3]{x}}{1+\sqrt[3]{x}}$. Then $f \in C([0, \infty),[0, \infty))$.

Let $\eta=\frac{1}{2}, r=1, R=8$. For $x \in[0, R]$, we have

$$
f(x) \leq f(R)=f(8)=\frac{70}{3}<24=3 R,
$$


and for $x \in[r, R]$, we have

$$
f(x) \geq f(r)=f(1)=\frac{1033}{64}>16=\frac{2 r}{\eta(1-\eta)^{2}} .
$$

Hence, by Theorem 2, BVP 4.1 has at least one monotone positive solution $x^{*}(t)$ such that

and

$$
2 t \leq x^{*}(t) \leq-4 t^{3}+12 t, \quad t \in\left[0, \frac{1}{2}\right],
$$

$$
-\frac{8}{3}\left(t-\frac{1}{2}\right)^{3}+2 t \leq x^{*}(t) \leq-4 t^{3}+12 t, \quad t \in\left[\frac{1}{2}, 1\right] .
$$

Remark 1. In this example, the function $f$ satisfy condition $f(0)=0$, thus the problem has a trivial solution.

\section{ACKNOWLEDGEMENT}

The authors express their gratitude to the referee for her/his careful reading of the paper and useful suggestions.

\section{REFERENCES}

[1] R. P. Agarwal, M. Bohner, and P. J. Wong, "Positive solutions and eigenvalues of conjugate boundary value problems," Proc. Edinb. Math. Soc., II. Ser., vol. 42, no. 2, pp. 349-374, 1999.

[2] R. P. Agarwal, D. O'Regan, and P. J. Y. Wong, Positive solutions of differential, difference and integral equations. Dordrecht: Kluwer Academic Publishers, 1999.

[3] D. Anderson, "Multiple positive solutions for a three-point boundary value problem," Math. Comput. Modelling, vol. 27, no. 6, pp. 49-57, 1998.

[4] D. Anderson and R. Avery, "Multiple positive solutions to a third-order discrete focal boundary value problem," Comput. Math. Appl., vol. 42, no. 3-5, pp. 333-340, 2001.

[5] D. R. Anderson and R. I. Avery, "Fixed point theorem of cone expansion and compression of functional type," J. Difference Equ. Appl., vol. 8, no. 11, pp. 1073-1083, 2002.

[6] R. I. Avery, D. R. Anderson, and R. J. Krueger, "An extension of the fixed point theorem of cone expansion and compression of functional type," Commun. Appl. Nonlinear Anal., vol. 13, no. 1, pp. 15-26, 2006.

[7] A. Cabada, "The method of lower and upper solutions for second, third, fourth, and higher order boundary value problems," J. Math. Anal. Appl., vol. 185, no. 2, pp. 302-320, 1994.

[8] A. Cabada, "The method of lower and upper solutions for third-order periodic boundary value problems," J. Math. Anal. Appl., vol. 195, no. 2, pp. 568-589, 1995.

[9] A. Cabada, M. do Rosário Grossinho, and F. Minhós, "On the solvability of some discontinuous third order nonlinear differential equations with two point boundary conditions," J. Math. Anal. Appl., vol. 285, no. 1, pp. 174-190, 2003.

[10] A. Cabada and S. Lois, "Existence of solution for discontinuous third order boundary value problems," J. Comput. Appl. Math., vol. 110, no. 1, pp. 105-114, 1999.

[11] M. do Rosário Grossinho and F. M. Minhós, "Existence result for some third order separated boundary value problems," Nonlinear Anal., Theory Methods Appl., Ser. A, Theory Methods, vol. 47, no. 4, pp. 2407-2418, 2001.

[12] J. E. Ehrke, "Positive solutions of a left focal second order boundary value problems," Commun. Appl. Nonlinear Anal., vol. 14, no. 2, pp. 57-65, 2007. 
[13] Y. Feng, "Solution and positive solution of a semilinear third-order equation," J. Appl. Math. Comput., vol. 29, no. 1-2, pp. 153-161, 2009.

[14] J. R. Graef and B. Yang, "Multiple positive solutions to a three point third order boundary value problem," Discrete Contin. Dyn. Syst., vol. 2005, pp. 337-344, 2005.

[15] J. R. Graef and B. Yang, "Positive solutions of a nonlinear third order eigenvalue problem," Dyn. Syst. Appl., vol. 15, no. 1, pp. 97-110, 2006.

[16] J. R. Graef and B. Yang, "Positive solutions of a higher order boundary value problem," J. Appl. Math. Comput., vol. 36, no. 1-2, pp. 319-331, 2011.

[17] M. Greguš, Third order linear differential equations. Transl. from the Slovak by J. Dravecký, ser. Mathematics and its Applications (East European Series). Dordrecht etc.: D. Reidel Publishing Company, a member of the Kluwer Academic Publishers Group. (Co-ed. with VEDA, Bratislava), 1987, vol. 22.

[18] M. Greguš, "Two sorts of boundary-value problems of nonlinear third order differential equations," Arch. Math., Brno, vol. 30, no. 4, pp. 285-292, 1994.

[19] D. Guo and V. Lakshmikantham, Nonlinear problems in abstract cones, ser. Notes and Reports in Mathematics in Science and Engineering. Boston: MA: Academic Press, Inc., 1988, vol. 5.

[20] Y. Guo, Y. Liu, and Y. Liang, "Positive solutions for the third-order boundary value problems with the second derivatives," Bound. Value Probl., vol. 2012, p. 9, 2012.

[21] S. Li, "Positive solutions of nonlinear singular third-order two-point boundary value problem." $J$. Math. Anal. Appl., vol. 323, no. 1, pp. 413-425, 2006.

[22] Z. Liu, J. S. Ume, D. R. Anderson, and S. M. Kang, "Twin monotone positive solutions to a singular nonlinear third-order differential equation," J. Math. Anal. Appl., vol. 334, no. 1, pp. 299-313, 2007.

[23] Z. Liu, J. S. Ume, and S. M. Kang, "Positive solutions of a singular nonlinear third order two-point boundary value problem," J. Math. Anal. Appl., vol. 326, no. 1, pp. 589-601, 2007.

[24] Y. Sun, "Positive solutions of singular third-order three-point boundary value problem," J. Math. Anal. Appl., vol. 306, no. 2, pp. 589-603, 2005.

[25] Y. Sun, "Existence and iteration of monotone positive solutions for a third-order two-point boundary value problem," Appl. Math., Ser. B (Engl. Ed.), vol. 23, no. 4, pp. 413-419, 2008.

[26] Q. Yao, "The existence and multipilicity of positive solutions for a third-order three-point boundary value problem," Acta Math. Appl. Sinica, vol. 19, pp. 117-122, 2003.

[27] K. Yosida, Functional analysis. 5th ed., ser. Grundlehren der Mathematischen Wissenschaften. Berlin-Heidelberg-New York: Springer-Verlag, 1978, vol. 123.

Authors' addresses

Yongping Sun

School of Electron and Information, Zhejiang University of Media and Communications, Hangzhou, Zhejiang 310018, China

E-mail address: sunyongping@126.com

\section{Min Zhao}

School of Electron and Information, Zhejiang University of Media and Communications, Hangzhou, Zhejiang 310018, China

E-mail address: zhaomin6660sina.com

\section{Shuhong Li}

Department of Mathematics, Lishui University, Lishui, Zhejiang 323000, China

E-mail address: mathlsh@126.com 